\title{
The research and application of virtual transformer substation roaming platform based on the OpenGVS
}

\author{
Liu Fang ${ }^{1}$;Li Hui ${ }^{1}$; \\ 1:School of Computer and Informaiton Technology, \\ Northeast university of petroleum;NEPU; \\ Daqing,Heilognjiang, 163318,China; \\ e-mail: lfliufang1983@126.com
}

\begin{abstract}
Roaming platform is the most important part of the virtual reality, with which can shorten the exploitation time to a great extend. This walkthrough platform is based on virtual transformer substation, and has added collision detect, mouse identifying, natural phenomenon simulation and some other key technologies. This system can basically come to the need of a walkthrough platform.
\end{abstract}

Keywords- Virtual Scene; Roaming Platform; Collision Detect; Mouse Picking

\section{INTRODUCTION}

Virtual reality is both an active technology research field and a collection of high and new technology[1], which includes computer graphics, multimedia technology, artificial intelligence, man-machine interface technology, sensor technology, highly parallel real-time computation technology and people's behavior technology and so on. In essence it is a kind of advanced computer user interface; various kinds of intuitive and natural real-time perception interactive means could provide user-friendly operation through to the user at the same time offer, such as sight and hearing and touch, etc.

Roaming platform has the most widely used in virtual reality system, construction roaming and simulation training and so on are all belong to roaming system application scope. Roaming platform includes visual database generation and general roaming engine, and the roaming engine is the core part of roaming platform. The roaming platform could extract the common function to realize code reuse, so can shorten the develop time, reduce waste of resources..

\section{THE RESEARCH OF ROAMING SYSTEM PLATFORM}

There are three kinds of roaming platform in our virtual reality research group.

\section{A. Building the roaming platform using MultiGen Creator combined with the OpenGVS.}

Creator is MultiGen - Paradigm Company's industry leading software tool set, used to produce high precision of real-time $3 \mathrm{~d}$ scene. Creator is a new generation of real-time simulation modeling software which could generate large lifelike scene in real-time under the premise of generation for simulation. The construction of the engine is in VC++environment using OpenGVS development. OpenGVS is a kind of based on real-time $3 \mathrm{~d}$ software system

\author{
Tong $\mathrm{Wei}^{2}$ \\ 2: Information center the first oil production company, \\ Daqing Oilfield; \\ Daqing,Heilognjiang 163318,China;
}

development of the senior application program interface (API), also a kind of advanced visual management software, which is the world's first general workstation platform 3d visual management software [2].

\section{B. Building the roaming platform using Virtools combined} with the 3DS Max.

Virtools is developed by the French global interactive 3d development solutions company VIRTOOLS, its 3d engine has become Microsoft Xbox recognition system. The characteristics of Virtools are easy to use, wide application field, short development process, affinity graphical interface, revealed in the visual effect is good. There are five optional modules in addition to their own 3d/VR development platform Virtools Dev, which are network Server Virtools Server, physical attribute module Physics Pack, artificial intelligence module AI Pack, Xbox development module and submerged platform VR Pack.

\section{Building the roaming platform using OpenGL.}

OpenGL is a standard application programming interface provided for 3d graphics, is an independent of hardware equipment[3], window system and operating system graphics and hardware interface, which is supported in many platform, the developer can use it to design a powerful efficient interactive graphics application program.

\section{Comparison conclusion}

We could come to the following conclusion through compare three kinds of platform.

(1) Visualization of Virtools is higher than other tools, the program design processes of OpenGVS and OpenGL are not visual, because they are both based on $\mathrm{VC}++$ graphical SDK. It is higher demand for personnel ability of programming using OpenGVS and OpenGL to develop program, it is also because of this, the function of penGVS and OpenGL are flexible powerful.

(2)There is no model constructing function in Virtools, the object and its action made in 3DS Max must be imported into Virtools to edit and integrate. The combination OpenGVS with MultiGen Creator is also made to overcome their shortcomings, so it is a good platform.

(3)The program design by Virtools is based on the function modules, so it is difficulty to change some bottom set, more difficult for users to consider from the bottom to optimize the whole application. using OpenGVS develops 
applications could not only directly call OpenGVS, but also could call bottom functions which are provided by not OpenGVS but VC++. OpenGL is used in environment $\mathrm{C}$ or $\mathrm{C}++$ languages, the procedure design is flexible; the network interface design is convenient. OpenGL more focus on three dimensional graphics base development, modeling, interactive and program design work, many advanced graphics transform functions such as LOD and so on are not provided, so need developers to design by themselves, which need more time and energy.

(4)The development cycle of Virtools is shorter than OpenGVS and OpenGL. The OpenGL programming is more complex and project cycle is longer, the human investment big, once the task is changed, it is difficult to do software modification, maintenance cost larger.

Table1 COMPARING THE THREE KINDS OF PLATFORM

\begin{tabular}{|c|c|c|c|c|}
\hline & $\begin{array}{c}\text { Development } \\
\text { cycle }\end{array}$ & scalability & visibility & $\begin{array}{c}\text { Programming } \\
\text { requirement }\end{array}$ \\
\hline Virtools & Short & General & Yes & Low \\
\hline OpenGVS & Moderate & Good & No & High \\
\hline $\begin{array}{c}\text { OpenG } \\
\text { L }\end{array}$ & Long & Good & No & High \\
\hline
\end{tabular}

Because the virtual transformer substation roaming system has many external expansion, so the combination of MultiGen and OpenGVS is chosen this is flexible and relatively easy to develop. Although the development cycle of using the above combination is longer than using Virtools, OpenGVS encapsulates many functions, which is useful to quickly complete the system. Own to base on $\mathrm{VC}++$, the flexibility of OpenGVS is better than Virtools, such as processing data and simulating some natural effects and the scalability is also very strong.

\section{CREATING SCENE DATABASE}

The models of the virtual transformer substation roaming system are constructed by MultiGen Creator. Scene database is saved as tree structure, which is complying with the modeling software structure. The virtual scene includes the static entity model and dynamic entity model, the static physical model is refers to the topography, geomorphology, houses, trees and so on static model; the dynamic entity model refers to all kinds of simulation entity model, such as automobile, airplane. The construction of the dynamic entity in the system uses the degree of freedom technology. The degree of freedom could be set in any mobile part of the whole model, which contains parameter variables related with rotation, scaling, and displacement, using it could control model GROUP attached in DOF mode (degree of freedom), so realize the single control in every part of the model, descript the state of motion object more accurately. The movement of the door and various switch operation in the system are all using the technology. As shown in figure 1.

\section{COLLISION DETECTION}

The users of virtual reality system hope that the virtual objects in the sense could be seem as" physical existence", the authenticity of the behavior is the necessary conditions of the guarantee of the immersive virtual roaming system [4], however the support for the function of collision detection is the necessary conditions of the guarantee of the authenticity of the behavior. There are a variety of collision detection methods; the goal of most people research polyhedron algorithm for detection of collision is to reduce amount of the edge and face for collision detection. The common first step of many collision detection methods is to use approximate boundary area (usually use axis aligned boundary box or boundary ball) cover test to quickly reduce not related many objects. As the extension of such kind of thought, layered boundary area method is used to rapidly determine the collision area. Another kind of collision detection method is tracking the distance of each pair of the object in the virtual world, if the distance between a pair of the object is less than a certain threshold, the objects collide each other.

Considering above collision detection methods and the special requirements of collision detection in the virtual roaming system, the forward line detection of collision detection method is adopted which is based on the line of sight. Using this method, three parameters (v_p0, v_p1, v_p2) used in collision detection are defined for Observation camera, v_p0 means the position of current camera; v_p1 means the position of a point, the distance between that and the current camera is L in the camera forward direction; v_p2 means the position of the other point, the distance between this point and the current camera is $\mathrm{L}$ in the camera back direction. The two space linear sections determined by the above parameters are responsible for the collision detection in the camera forward and backward movement [5].

Using the collision detection algorithm based on the line of sight, the most important operation is how to quickly determine the collision generation. In the implementation, first using function GV_cam_inq_position() to get the position of the current camera in the world, the camera view vector could be get by using function(). Secondly, making a line segment in the forward of the return point, so there are two coordinate points pos 1 and pos2, the distance between the two coordinate points is L.Along with the movement of the camera, the position of the line will change, using function GV_geo_inq_intersection( scene, \&pos1, \&pos2, isc_mask, EXCLUDED, \&isc_data) to do the intersection operation, whether the motion object and the scene objects is intersection is judged by pos 1 point and pos 2 point are between the both sides of a face. If intersecting, the object will stop moving until other orientation movement happens, parameter isc_mask means the element detected in the collision, include face, sub object and so on; parameter EXCLUDED means the objects which could be ignored during the testing; whether the Collision is occurring could be judged by the returned Boolean variable parameters isc_data.isc_resul. 


\section{SimUlating NATURAL EFFECTS}

The natural effects in this system mainly includes the simulation of the sun (time simulation, halation simulation), the clouds simulation, raindrop simulation, flame simulation, smoke simulation, water simulation and so on. The method of simulating natural effects is always using particle system [6]. With the passage of time, the system not only has some particle changing shape, in constant motion, but also has new particle to join, old particle constantly disappear. In order to simulate the particle growth and death process, each particle all have certain life cycle, birth, growth, aging and death process. In every moment (each frame) only part of it is "existence", therefore, in the applications of particle system, the law of the birth and death of the particle must be in a certain extent, and must conform to the object modeling of physical laws. The general structure of the particle system is shown in figure 2 .

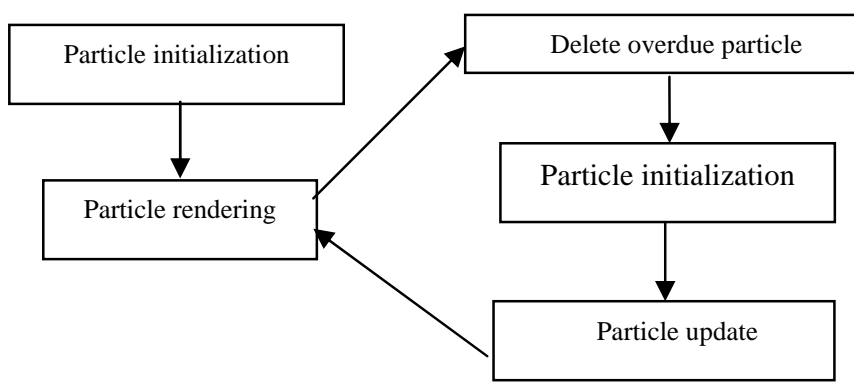

Figure 2. The general structure of the particle system

The function GVU_isg_( ) and GVU_smk_( ) supplied by OpenGVS ${ }^{[4]}$ are used in the implementation, first using function GV_obd_0pen_by_name( ) to define effect object; secondly,using graphics callback function GV_obd_set_gfx_callback( ) and a series of function to initialize the control parameters of the display list ;finally, using GV_obi_jnstance( ) to instance the object.Through simulating natural effects, how to work in bad weather condition and the matters need to attention can be truly shown. The simulation effects are shown as follow.
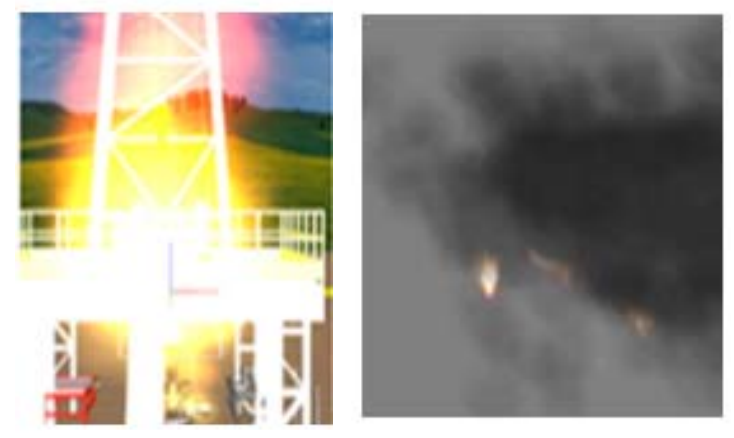

Figure 3. Flame and smoke simulation rendering
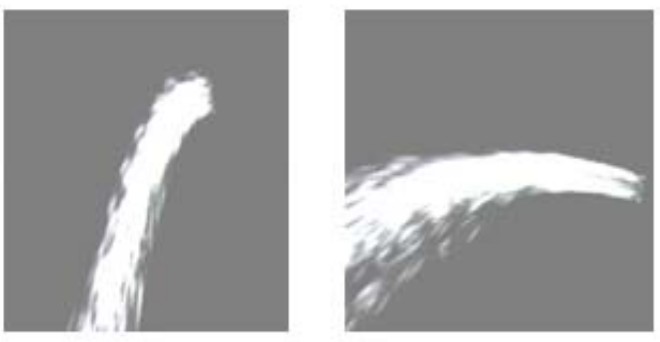

Figure 4. Flow simulation rendering

\section{MOUSE PICKING}

Virtual environment should fully embody the manmachine interactive; the observers can't like seeing the movies, but can really participate in activities in virtual environment, and could interact with virtual environment. For example, in a building of virtual environment, when the Roaming Walkers use the mouse to click the room door knob, door automatically open, or use the mouse to click the simulation scene of substation operation equipment, the equipment should be able to complete the corresponding action. The mouse picking function are used to solve these question. The mouse picking function in this system is based on ray detection of collision detection method, according to the matrix used in virtual environment do the inverse transform to projection ray and intersect with the model to pick up objects, this method can save a large amount of computing time and access space. The process is that first using mouse chick somewhere on the screen, the screen coordinates clicked by mouse are arrived; secondly, through the visual area transformation getting projection point coordinates, then getting a ray started from view point go through the projection point; finally, do projection transformation to this ray and observe the transformation in the scene, if intersected with the model, the model is picked by the mouse.

\section{TWO-DIMENSIONAL MAP NAVIGATION}

Two-dimensional map is an essential part of roaming platform. Through the two dimensional map, users could observe the scene panorama more intuitive and understand their position commodiously. Two-dimensional map in the roaming platform is realized by using OpenGVS double channel mechanism. By using a look down camera observe the location of the wanderer at any time to accurate positioning, by clicking on the map; the visitors can quickly switch to this position. As shown in figure 5. 


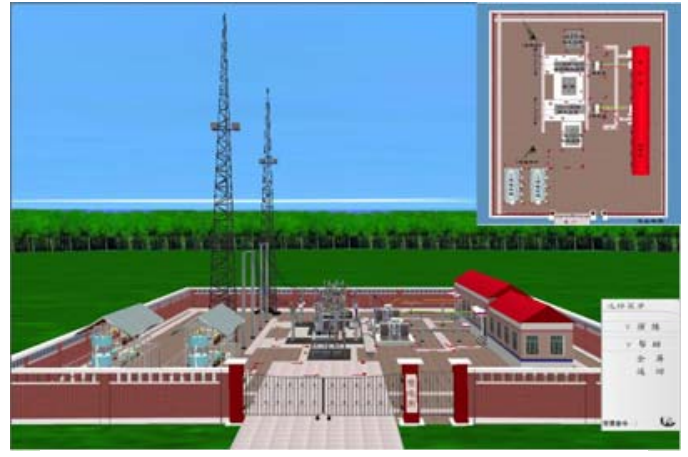

Figure 5. Two-dimensional navigation map

\section{CONCLUSION}

Virtual construction roaming system is a comprehensive application of virtual reality technology, which can be used for public construction projects such as station, the dam and power station, urban planning design, building interior design, etc.The application of roaming platform could directly show the architectural scene integral design, performance, feasibility planning, could reduce design phase error, could not only ensure construction quality but also save money to improve the efficiency of design and review.With the roaming technology constantly mature, its applications will have a more broad prospects and more social and economic benefit.

\section{REFERENCES}

[1] TUrnerAK.Three-Dimensional Modeling with Geoseientifie Information Systems [M].NATO KluwerAeademiePublishers, Dordrecht.1992.

[2] OpenGVS Programming Guide[J] ( Version 4.3) . Quantum3D. Inc. and Gemini Technology Corporation, 1999 : 23-30.

[3] WRIGHT,Jr,R S,SWEET M.OpenGL Super Bible[M].2nd edition.Corte Madera,California:Waite Group Press,1999

[4] W. A. Badawy, H. M. Kelash. Collision Detection in VRML: A Survey [J]. EmiratesJournal for Engineering Research, 2003, 8(2): $1-8$.

[5] John Ketchel, Pierre Larochelle. Collision Detection of Cylindrical Rigid Bodies forMotion Planning [A]. Proceedings of the 2006 IEEE International Conference on Robotics and Automation, 2006, 15301535.

[6] Reeves W. T. Particle System-A Technique for Modeling A Class of Fuzzy Objects [J]. Computer Graphics, 1983, 17(2):359 376. 